\title{
MODIS AOT Based Inverse Modeling for Asian Dust
}

\author{
Keiya Yumimoto ${ }^{1}$, Itsushi Uno ${ }^{1}$, Nobuo Sugimoto², and Atsushi Shimizu ${ }^{2}$ \\ ${ }^{1}$ Research Institute for Applied Mechanics, Kyushu University, Fukuoka, Japan \\ ${ }^{2}$ National Institute for Environmental Study, Tsukuba, Japan
}

\begin{abstract}
Inverse modeling of Asian dust was performed using MODIS coarse-mode aerosol optical thickness (AOT) and a regional adjoint dust model. The a posteriori AOT shows better agreement with the MODIS AOT and OMI Aerosol index (AI). Independent validation with the NIES Lidar network shows that the inversion leads to a significant improvement and reduces root mean squares of differences by $29-32 \%$ at Seoul, Matsue, and Toyama. The inversion results show considerably greater dust emissions over the Gobi Desert and Mongolia. Especially, optimized emissions are estimated as $8.1 \mathrm{Tg}$ (352\% of the a priori) for 29 March and $19.5 \mathrm{Tg}$ (325\%) for 30 March. Comparison with inversion results using NIES Lidar (Yumimoto et al. 2008) shows consistency with our a posteriori dust emissions which underscores the feasibility of dust inversion with satellite optical observations, and encourages combined inversion that assimilates the Lidar extinction coefficient (vertical profiles) and MODIS AOT (horizontal distribution) concurrently. However, the a posteriori results might reflect effects of air pollutants from highly industrialized regions, which might contaminate the assimilated results. Further quality control of the input for the data assimilation is necessary in future studies.
\end{abstract}

\section{Introduction}

During the last decade, several hindcast analyses and predictions of Asian dust were performed using numerical models (e.g., Uno et al. 2004). These analyses revealed various characteristics of Asian dust outflows in conjunction with observation data. However, great uncertainty remains in the dust numerical models. Uno et al. (2006) completed the Dust Model Inter-comparison project (DMIP), which showed that dust emission fluxes estimated using eight different models were 27-336 Tg for March 2002 and 18-103 Tg during April 2002. Surface dust concentrations sometimes differed by 10 times or more at Beijing. This fact reflects that variability in dust emission dominates the model uncertainty.

Yumimoto et al. $(2007,2008)$ developed a regional dust transport model with a four-dimensional variational (4D-Var) data assimilation system, with inversely optimized Asian dust emission data using the NIES Lidar observation network (Sugimoto et al. 2006). Although lidar observations provide dust extinction coefficients with high time and vertical resolutions (15 min and $30 \mathrm{~m}$, respectively) and are already networked over eastern Asia, lidar networks have difficulty capturing detailed horizontal distributions of dust outflows. In contrast, recent satellite observations (e.g., ModerateResolution Imaging Spectroradiometry (MODIS) and Multiangle Imaging Spectroradiometry (MISR)) can capture horizontal distributions of aerosols quantitatively by measuring their optical characteristics. Recently, Dubovik et al. (2008) developed an algorithm for retrieving aerosol sources from MODIS Aerosol optical Thickness (AOT) by inversion modeling with the GOCART model. Zhang et al. (2008) assimilated MODIS AOT with their operational forecasting system (NAAPS) using a two-dimensional variational method. However,

Corresponding author: Keiya Yumimoto, Kasuga Park 6-1, Kasuga, Fukuoka 816-8580, Japan. E-mail: yumimoto@riam. kyushu-u.ac.jp. @2008, the Meteorological Society of Japan. assimilation and inverse modeling of aerosols data using satellite observations remain limited; they are not well evaluated yet. For this study, we perform inverse modeling of Asian dust using AOT measured using a MODIS onboard NASA/TERRA and AQUA with a regional data assimilation system. We thereby demonstrate the feasibility of the inversion. To our knowledge, this is the first inversion modeling of Asian dust using satellite observations and a regional adjoint dust model.

\section{Numerical experiment setup and observa- tion}

\subsection{Assimilated dust emission/transport model}

The RAMS/CFORS-4DVAR (RC4; Yumimoto et al. 2007, 2008) is a 4D-Var chemical data assimilation system based on a regional chemical transport model: the Chemical FORcasting System (RAMS/CFORS; Uno et al. 2004). In fact, RC4 has been used for adjoint inversions of CO emission (Tanimoto et al. 2008) and Asian dust outflow (Uno et al. 2008; Hara et al. 2008). Herein, we provide a brief description of only the latest updated points of the system. Yumimoto et al. (2008) describe additional details. In RC4, the dust emission flux is calculated as

$$
F_{k}=f\left(\varepsilon_{i j}\right) C_{i j} u_{*}^{3}\left(u_{*}-u_{*, t h}\right)
$$

where $C$ represents a dimensional coefficient depending upon surface information (e.g., soil moisture); the $i j$ suffix denotes a grid point. Respectively, $u_{*}$ and $u_{*}$, th denote the surface friction velocity and threshold friction velocity. In addition, $\varepsilon$ is a control parameter; it is adjusted to optimize the dust emission flux. The value of $f(\varepsilon)$ in Eq. (1) is given as follows.

$$
f(\varepsilon)=\frac{20}{1+\exp \{0.2(15.72-\varepsilon)\}} .
$$

Equation (2) represents a sigmoid function with two asymptotic lines of 0 and 20. The greatest advantage of using this function is that the two asymptotic lines prevent the emission flux from being assigned an extremely large or negative value in the process of inversion.

For this study, we define the cost function as the following.

$$
\begin{aligned}
f(\varepsilon)= & \frac{1}{2}\left(\varepsilon-\varepsilon_{b}\right)^{T} \mathbf{B}^{-1}\left(\varepsilon-\varepsilon_{b}\right)+\frac{1}{2} \sum\left(H(\varepsilon)-\tau_{\text {coarse }}\right)^{T} \mathbf{R}^{-1}\left(H(\varepsilon)-\tau_{\text {coarse }}\right) \\
& +\frac{\gamma}{2}\|\Delta(\varepsilon-1)\|^{2} .
\end{aligned}
$$

In that equation, $\tau_{\text {coarse }}$ and $\mathbf{R}$ represent MODIS coarsemode AOT observations and an observation error, as described in the following subsection. The third term represents a smoothing term. $H$ is the observation operator including the forward CFORS model. In this study, we assimilate horizontal distributions of MODIS $\operatorname{AOT}\left(\tau_{\text {coarse }}\right)$, optimize $\varepsilon$ daily at each grid point, and obtain a posteriori dust emissions and assimilated dust concentrations. The background error of the dust emission flux (B) is assigned 500\%, referring to results of DMIP. In RC4, an iterative optimization routine is used to minimize the cost function, and 16 iterations were performed to obtain optimal solutions.

The RC4 domain is centered at $37.5^{\circ} \mathrm{N}$ and $115^{\circ} \mathrm{E}$ on a rotated polar stereographic system (Fig. 1a). The horizontal grid comprises $180 \times 100$ grid points with resolution of $40 \mathrm{~km}$. Vertical layers extend from the surface to 


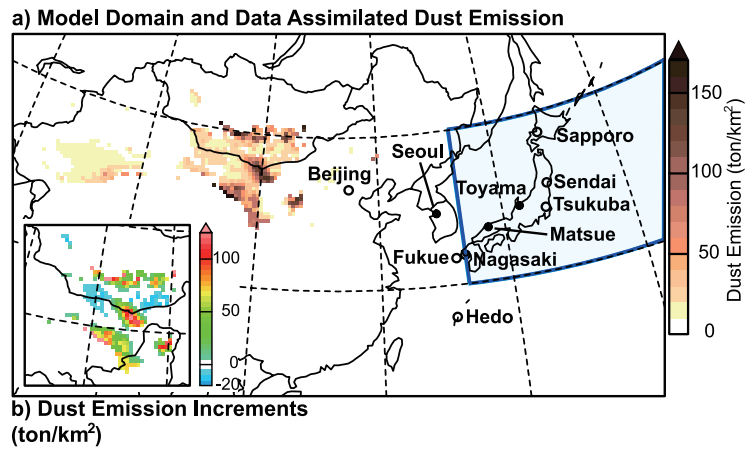

Fig. 1. Model domain, NIES Lidar observation sites (solid circle points are used for model validation) and the MODIS data region used for inversion: (a) a posteriori dust emission; (b) increment between a priori and a posteriori dust emissions.

$23 \mathrm{~km}$ with 40 stretching grid layers. Actually, RC4 uses meteorological fields provided using a meso-scale meteorological model RAMS nudged by NCEP reanalysis $(2.5$ $\times 2.5 \mathrm{deg}$ ). For this study, we targeted the dust event that occurred over the Gobi Desert during 29-30 March 2007 and hit Japan's islands during 30 March and 1 April (Yumimoto et al. 2008). The assimilation window (simulation period) was from 28 March to 3 April 2007.

\subsection{Observation data}

We use a coarse mode AOT $\left(\tau_{\text {coarse }}\right)$ derived from "the aerosol optical depth" and "the aerosol optical depth ratio small" provided by Level-3 MODIS Atmosphere Daily Global Product (Remer et al. 2005: http://modisatmos.gsfc.nasa.gov/MOD08_D3/index.html). We assume that the coarse mode particles are dominated by soil dust and maritime aerosols (Dubovik et al. 2008). Only those AOT observations made over the Sea of Japan and the western Pacific near Japan (shown by the blue box in Fig. 1a) are used for the inversion. Coarse mode AOT is sometimes sensitive to anthropogenic aerosols near highly industrialized areas throughout eastern China (e.g., 31 March in Fig. 2). Therefore, we excluded satellite observation data over the East China Sea from the input to inversion. Moreover, MODIS AOT data have different accuracy over the ocean and over a land surface because of the difference in reflectance or albedo (AOT over land is more uncertain than that over ocean; Dubovik et al. 2008). Therefore, we assimilate the MODIS coarse mode AOT only over the ocean surface that is measured by TERRA at 02UTC (TERRA overpasses at 10:30 local time), and AQUA at 04UTC (AQUA overpasses 13:30 at local time) on 1 and 2 April. Consequently, four snapshots of MODIS coarse-mode AOT over the ocean are used for the inversion.

Remer et al. (2005) estimated the expected accuracy of MODIS AOT as $0.03 \pm 0.05 \tau$, where $\tau$ denotes MODIS AOT, over ocean. Coarse mode AOT is sensitive not only to dust but also to sea salt aerosols. Satake et al. (2004) studied the transport of the major tropospheric aerosols in eastern Asia during spring 2001 using the CFORS model, and estimated that averaged contributions of dust and sea salt to the total AOT are, respectively, 36.6 and 5.8\% over the Sea of Japan (the averaged relative contribution of sea salt to the sum of dust and sea salt aerosols, i.e., the coarse mode aerosol, is 14\%). This contribution ratio of sea salt aerosol is added into the relative observation error as an additional uncertainty. We also found that the setting of small observation error during the adjoint inversion sometimes produces an inadequate solution: in extreme cases, too small an error assigned by small AOT observation results in a numerical divergence. We assume a larger baseline absolute observation error (0.2) to prevent numerical instability. Consequently, we assume the observation error to be uncorrelated, and assign $0.2+(0.05+0.14)$ $\tau_{\text {coarse. }}$

\section{Results}

Figure 2 shows respective horizontal distributions of observed and modeled AOTs during 30-31 March and 1-2 April. The AURA/OMI Aerosol Index (AI) is also shown as an independent observation for model validation. The AOT measurements of 30 and 31 March are not used for the inversion. The OMI AI data for April 1 are missing.

On 30 March (Fig. 2: the top row), a heavy dust storm was caused by strong surface winds associated with a low-pressure area centered in Mongolia. The a posteriori results show increased dust concentrations and can capture two heavy dust loadings near the Gobi Desert measured by OMI AI, which the a priori simulation cannot reproduce (Fig. 2c: D1). Because of missing MODIS data, the root mean squares of differences (RMSD) between MODIS and model AOT show no improvement between a priori and a posteriori.

The dense dust loadings region (AOT $>2$ and $\mathrm{AI}>5$ ) moved eastward and reached northeastern China on 31 March (Fig. 2: the 2nd row). The a priori results can generally capture the horizontal distribution of the dense dust well, but they underestimate dust concentrations. The inversion emphasizes the AOT level considerably and engenders a reduction of the RMSD (17\%); MODIS AOT data observed on this day are not used for the inversion.

The next day (1 April: the third row of Fig. 2), the dust loading traveled toward the Sea of Japan. The inversion increases the a posteriori dust concentration and leads better agreement with MODIS AOT reducing the RMSD by $20 \%$. On 2 April (Fig. 2: the bottom row), both MODIS and OMI AI observations showed that the dust covered the islands of Japan extensively. The a posteriori emission improves underestimation of dust AOT over the islands of Japan, and provides better agreement with observations. The RMSD is reduced by $26 \%$. However, the inversion also emphasizes dust loading over southern China (Fig. 2c: D2), where OMI AI detects no heavy absorbing aerosol loading. A possible reason for this unrealistic AOT is discussed in a later section of this paper.

Validation using an independent observation set is crucially important. Figure 3 shows a comparison of NIES Lidar and a posteriori dust extinction coefficients. The NIES Lidar captured a heavy dust layer hit at Seoul on 31 March; then it reached Japanese sites (i.e., Matsue and Toyama) on 1 April with ca. $2 \mathrm{~km}$ depth. The a posteriori extinction coefficients agree quite well with observations at each lidar site, capturing onset times and structures of the dust layer.

To validate the inversion results more quantitatively, we calculate dust AOT by integration of the dust extinction coefficient under $6 \mathrm{~km}$. The bottom row of Fig. 3 shows a time series of lidar and RC4 dust AOT. This figure also includes the MODIS AOT observation near the stations (open circles indicate data not used for assimilation; solid circles show data used for assimilation). The a priori AOT generally reproduces the onset time, but it underestimates the observed AOT considerably. The inversion brings the modeled AOTs much closer to the observations at each Lidar site. The RMSD between the lidar and model dust AOT are reduced by 29-32\%.

Yumimoto et al. (2008) performed adjoint inversions using NIES Lidar observations (from their study, we referred a result for their Experiment A, which used Japanese lidar observation sites at Hedo-Okinawa, Nagasaki, Matsue, Toyama, and Tsukuba) for the same dust event (hereinafter designated as lidar inversion). The lidar inversion window is longer than that of the current MODIS study. Lidar inversion results are also 

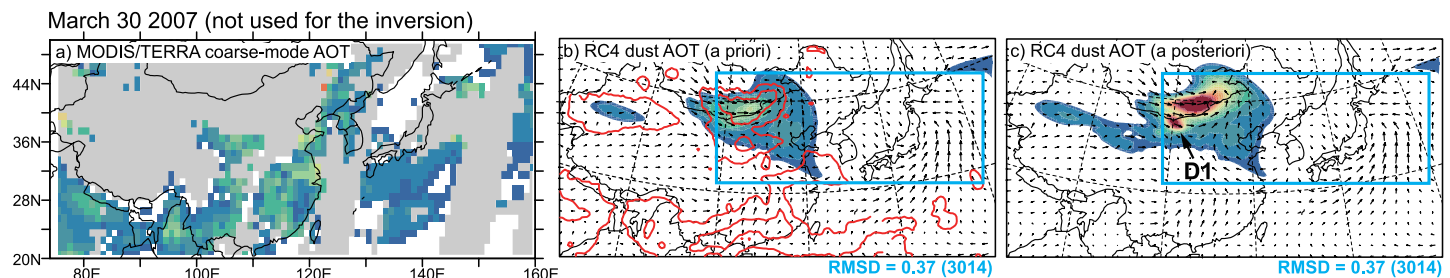

March 312007 (not used for the inversion)
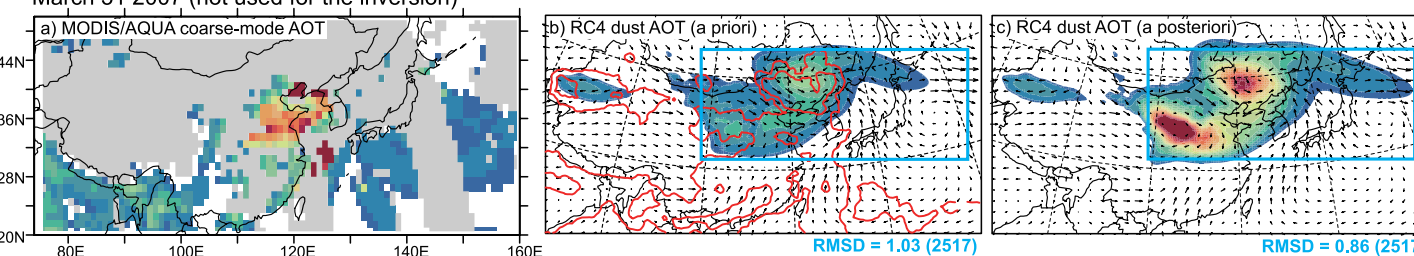

April 12007 (assimilated)
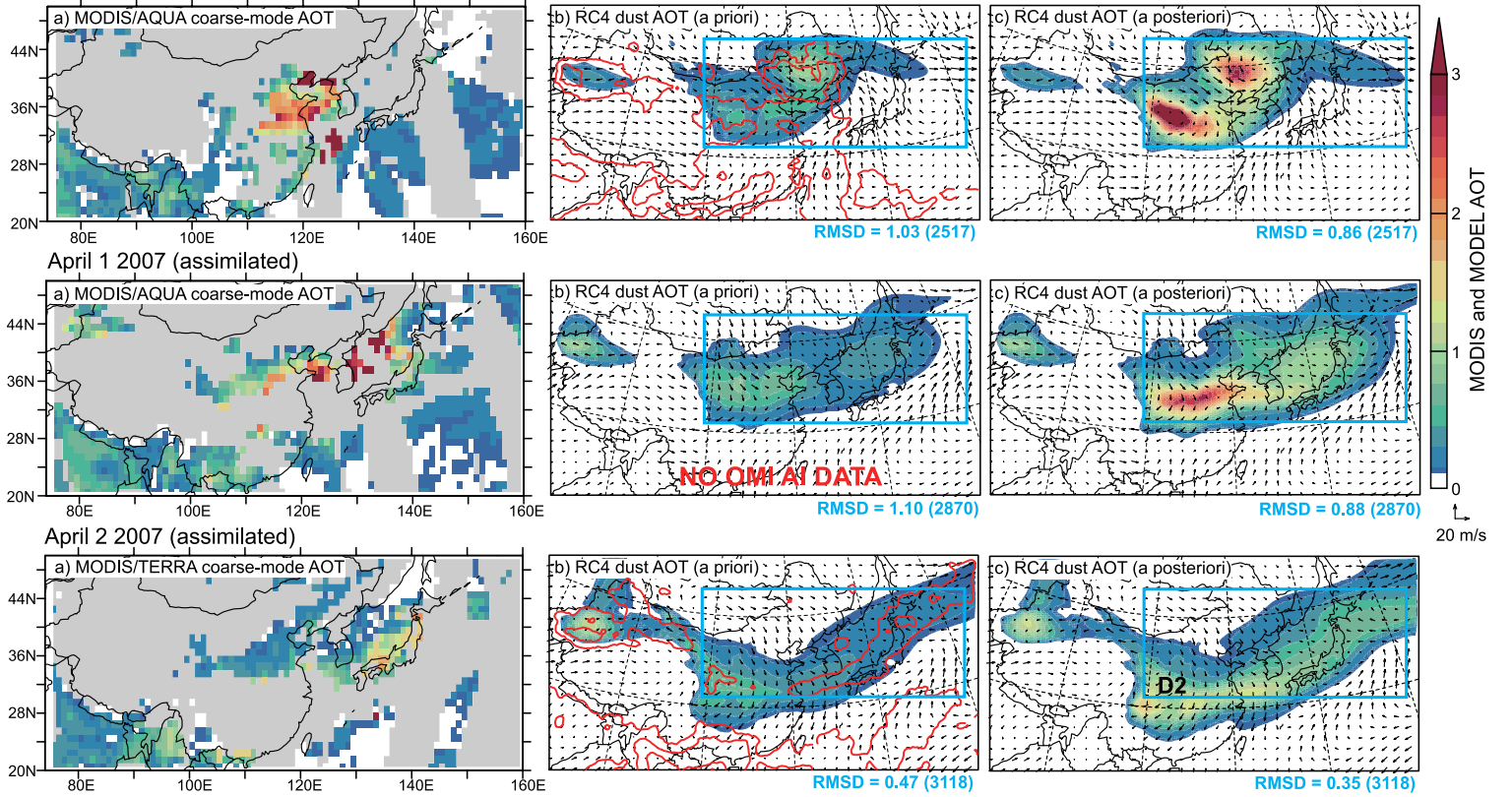

Fig. 2. Comparison of modeled AOT and satellite observations on 30-31 March and 1-2 April: (a) MODIS coarse mode AOT; (b) the a priori modeled dust AOT; (c) the a posteriori modeled dust AOT. AURA/OMI AI is shown as a red line (interval = 1, 3, 5). Grayedout areas in the left-most column show missing data. The root mean square difference (RMSD) between MODIS and modeled AOT in the region indicated by the blue box is also shown. Numbers in parentheses denote numbers of data used for RMSD calculation.

represented in the third row of Fig. 3. At Seoul and Toyama, our inversion partially underestimates the dust concentration compared to observations and the inversion using lidar (e.g., around noon on 31 March at Seoul). However, current results show overall good agreement with lidar inversion.

The results showing good agreement of MODIS inversion and lidar inversion are not surprising because both observations gave almost the same signal to the adjoint inversion system: they show almost equivalent dust onset timing and AOT level; see Fig. 3, third row. However, lidar inversion used the observed vertical profile. It therefore lends the possibility of correction of the modeled vertical profile, although MODIS AOT inversion has no such potential.

Figure 1a shows the a posteriori dust emission flux accumulated during the simulation period. Figure $1 \mathrm{~b}$ shows the increment of accumulated dust emissions between the a posteriori and the a priori values. The inversion estimates total Asian dust emission during 28 March and 3 April 2007 as 45.2 Tg, and engenders a significant increase (177\% of a priori): especially on 29 and 30 March, the a posteriori daily dust emissions are increased respectively to 8.1 (352\% of the a priori) and 19.5 Tg (325\%). The lidar inversion optimized the daily Asian dust emissions to 6.8-9.8 Tg for 29 March and 15.6-20.4 Tg for 30 March. Judging from Fig. 1b, dust emissions over the Gobi Desert and Mongolia are increased considerably. Our increment closely resembles that by the lidar inversion. These facts indicate that the inversion by satellite data captures the dust event and optimizes the a posteriori emission adequately, even if it does not provide a vertical dust profile.

Despite the good similarity, our increment is some- what higher than that by lidar observations over the southern Gobi desert (south of $40^{\circ} \mathrm{N}$ ). The assimilated data (e.g., AOT observations on 1 April) might include non-dust aerosols (e.g., air pollution from the highly industrialized region near Shanghai: see Fig. 2), and engender this higher increment over southern region of the Gobi desert through inversion. The high a posteriori AOT over southern China on 2 April (Fig. 2c: D2) might reflect this increase of dust emissions in the southern area. Zhang et al. (2008) introduced additional quality control (QC) and quality assurance (QA) into MODIS AOT products, and excluded outliers in their 2D variational assimilation system. Further initial quality control of observations must be explored in a future study.

\section{Conclusion}

We present results of an adjoint inversion of Asian dust outflow with MODIS coarse mode AOT targeting the dust episode during 29 March-2 April 2007.

The inversion results emphasize model dust AOT and bring the a posteriori results closer to those of MODIS AOT and OMI AI. The a posteriori results reproduced the two dense dust loadings over the dust source region, which the a priori cannot capture well. Independent validation was performed using the NIES Lidar network. The a posteriori dust extinction coefficients agree well with those observed using lidar, and show a significant improvement. The RMS differences (RMSD) between lidar and model dust AOT are lower by $29-32 \%$.

Inversion modeling increases dust emissions over the Gobi Desert and Mongolia: Especially on 29-30 

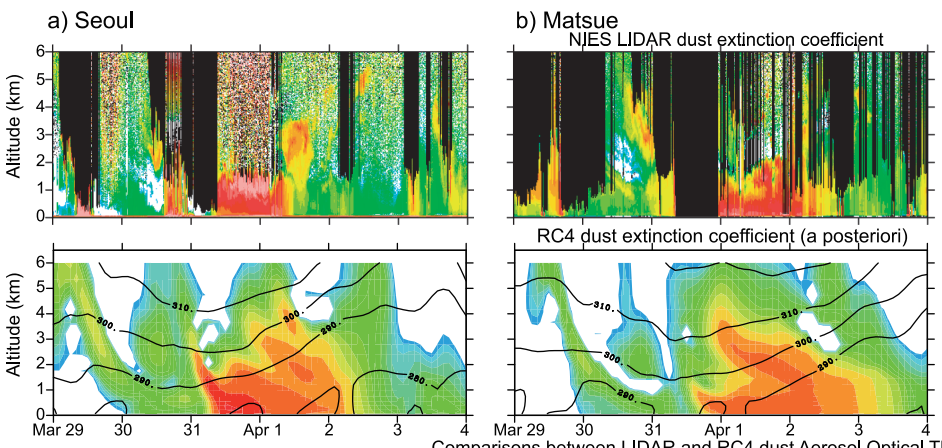

c) Toyama

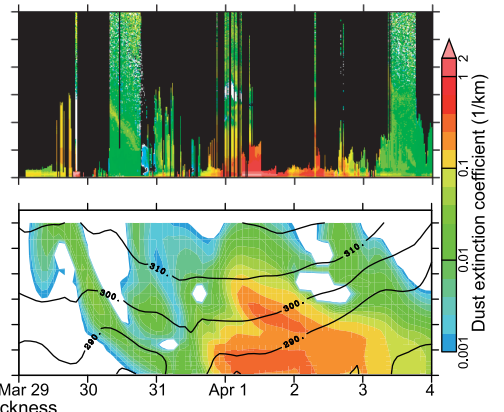

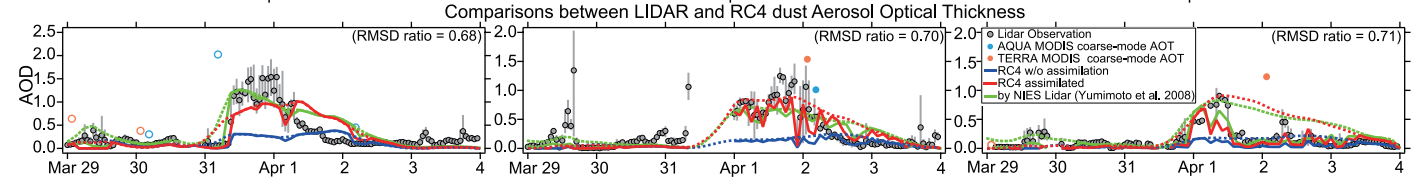

Fig. 3. Comparison of lidar-observed and modeled dust extinction coefficients and AOT at (a) Seoul, (b) Matsue, and (c) Toyama. The first row shows the Time-Height cross-section by the NIES Lidar. Blacked-out areas show missing data because of rain and clouds. The second row is the same as the first row, but with a posteriori model results. The third row shows the time series of lidar and modeled dust AOT. The dust AOT is calculated for altitudes of less than $6 \mathrm{~km}$. Solid lines show the AOT calculated by excluding the missing lidar observation points (blacked-out areas in top row). Dashed lines denote the AOT calculated using all vertical grid points. Inversion results obtained through NIES Lidar inversion (Yumimoto et al. 2008) are also presented as green lines. The ratio of the a priori by the a posteriori RMSD is also shown in numbers.

March, when a low-pressure front passed and its associated strong wind swept over, dust emissions increased considerably. The inversion estimates the dust emissions as $8.1 \mathrm{Tg}$ (352\% of the a priori) for 29 March and $19.5 \mathrm{Tg}(325 \%)$ for $30 \mathrm{March}$. The a posteriori dust emissions consist of that obtained by inverse modeling with NIES Lidar (Yumimoto et al. 2008), proving the feasibility of inversion for Asian dust using optical observations measured by satellites. However, over the southern Gobi region (south of $40^{\circ} \mathrm{N}$ : the Tegger Desert), our inversion shows a larger increment of dust emission than that found by Yumimito et al. (2008). Misclassified coarse-mode AOT information from non-dust aerosol particles (e.g., anthropogenic aerosol emitted from the highly industrialized regions of eastern China) might contaminate the inversion results and engender overprediction of the a posteriori AOT over southern China on 2 April. Additional quality control (QC) will improve the inversion and assimilation capability in the next step of this research. The consistency between the inversion results also underscores that the combined inversion, which simultaneously assimilates observations provided by lidar and satellite platforms, is promising for additional improvement of the dust adjoint modeling.

\section{Acknowledgments}

This work was partly supported by the Global Environment Research Fund, Ministry of Environment, Japan (C-061).

\section{References}

Dubovik, O., T. Lapyonok, Y. J. Kaufman, M. Chin, P. Ginoux, R. A. Kahn, and A. Sinyuk, 2008: Retrieving global aerosol sources from satellites using inverse modeling. Atmos. Chem. Phys., 8, 209-250.

Hara, Y., K. Yumimoto, I. Uno, A. Shimizu, N. Sugimoto, Z. Liu, and D. M. Winker, 2008: Asian dust outflow in the PBL and free atmosphere retrieved by NASA CALIPSO and an assimilated dust transport model. Atmos. Chem. Phys. Discuss., 8, 8715-8742.

Remer, L. A., Y. J. Kaufman, D. Tanre, S. Mattoo, D. A. Chu, J. V. Martins, R.-R. Li, C. Ichoku, R. C. Levy, R. G. Kleidman, T. F. Eck, E. Vermote, and B. N. Holben, 2005: The MODIS Aerosol Algorithm, Products and Validation. J. Atmos. Sci., 62, 947-973.
Satake, S., I. Uno, T. Takemura, G. R. Carmichael, Y. Tang, D. Streets, N. Sugimoto, A. Shimizu, M. Uematsu, J.-S. Han, and S. Ohta, 2004: Characteristics of Asian aerosol transport simulated with a regional-scale chemical transport model during the ACE-Asia observation. J. Geophys. Res., 109, D19S22, doi:10.1029/2003JD003997.

Sugimoto, N., A. Shimizu, I. Matsui, X. Dong, J. Zhou, X. Bai, J. Zhou, C.-H. Lee, S.-H. Yoon, H. Okamoto, and I. Uno, 2006: Network observations of Asian dust and air pollution aerosols using two-wavelength polarization lidars, $23^{\text {rd }}$ International Laser Rader Conference, July 2006 Nara, Japan, (23ILRC, ISBN 4-9902916-0-3), 851-854.

Tanimoto, H., Y. Sawa, S. Yonemura, K. Yumimoto, H. Matsueda, I. Uno, T. Hayasaka, H. Mukai, Y. Tohjima, K. Tsuboi, and L. Zhang, 2008: Diagnosing recent CO emissions and ozone evolution in East Asia using coordinated surface observations, adjoint inverse modeling, and MOPITT satellite data, Atmos. Chem. Phys., 8, 3867-3880.

Uno, I., S. Satake, G. R. Carmichael, Y. Tang, Z. Wang, T. Takemura, N. Sugimoto, A. Shimizu, T. Murayama, T. A. Cahill, S. Cliff, M. Uematsu, S. Ohta, P. K. Quinn, and T. S. Bates, 2004: Numerical study of Asian dust transport during the springtime of 2001 simulated with the Chemical Weather Forecasting System (CFORS) model. J. Geophys. Res., 109, D19S24, doi: 10.1029/2003JD004222.

Uno, I., Z. Wang, M. Chiba, Y. S. Chun, S. L. Gong, Y. Hara, E. Jung, S.-S. Lee, M. Liu, M. Mikami, S. Music, S. Nickovic, S. Satake, Y. Shao, Z. Song, N. Sugimoto, T. Tanaka, and D. L. Westphal, 2006: Dust model intercomparison (DMIP) study over Asia - Overview. J. Geophys. Res., 111, D12213, doi:10.1029/2005JD006575.

Uno, I., K. Yumimoto, A. Shimizu, Y. Hara, N. Sugimoto, Z. Wang, Z. Liu, and D. M. Winker, 2008: 3D structure of Asian dust transport revealed by CALIPSO lidar and a 4DVAR dust model. Geophys. Res. Lett., 35, doi:10.1029/ 2007 GL032329.

Yumimoto, K., I. Uno, N. Sugimoto, A. Shimizu, and S. Satake 2007: Adjoint inversion modelling of dust emission and transport over East Asia. Geophys. Res. Lett., 34, L08806, doi:10.1029/2006GL028551.

Yumimoto, K., I. Uno, N. Sugimoto, A. Shimizu, Z. Liu, and D. M. Winker, 2008: Adjoint inversion modeling of Asian dust emission using lidar observations. Atmos. Chem. Phys., 8, 2869-2884.

Zhang, J., J. S. Reid, D. L. Westphal, N. L. Baker, and E. J. Hyer, 2008: A system for operational aerosol optical depth data assimilation over global oceans. J. Geophys. Res., 113, D10208, doi:10.1029/2007JD009065.

Manuscript received 16 July 2008, accepted 3 September 2008 SOLA: http://www.jstage.jst.go.jp/browse/sola/ 